\title{
Comparando pobreza laboral: Los casos de Chile y España
}

\section{Comparing labor poverty: The cases of Chille and Spain}

\author{
José Zawadsky \\ joseeduardo.zawadsky@e-campus.uab.cat \\ Universitat Autònoma de Barcelona, España
}

Recepción: 20 Mayo 2020

Aprobación: 12 Octubre 2020

Publicación: 01 Febrero 2021

Cita sugerida: Zawadsky, J. (2021). Comparando pobreza laboral: Los casos de Chile y España. Cuestiones de Sociología, 24, e110. https://doi.org/10.24215/23468904e110
Resumen: Esta contribución realiza un análisis comparado de la pobreza laboral en Chile y España, dando cuenta de cómo un grupo de trabajadores pertenecientes principalmente al segmento secundario forman parte de hogares en situación de pobreza. Luego de una breve introducción, revisamos la literatura respecto de la temática para conocer las principales dimensiones que inciden en la pobreza laboral. En el tercer apartado contextualizaremos los casos de estudio, para luego conocer las características y la vivencia de la pobreza laboral en ambos casos. Finalmente, identificamos las principales semejanzas y diferencias: mientras para ambos casos encontramos similares características de la situación ocupacional asociadas a la pobreza laboral, en el área de la experiencia encontramos diferencias. El estudio comparado de la pobreza laboral nos permite comprender de mejor manera los orígenes de la misma y sus diversas consecuencias, con miras a superar las discusiones sobre casos particulares y contribuir a la creación de un marco analítico general.

Palabras clave: Pobreza laboral, Trabajadores pobres.

Abstract: This contribution makes a comparative analysis of working poverty in Chile and Spain, showing how a group of workers belonging mainly to the secondary segment are part of households living in poverty. After a brief introduction, we review the literature on the subject to learn about the main dimensions that affect working poverty. In the third section we will contextualize the case studies, and then learn about the characteristics and experience of working poverty in both cases. Finally, we identify the main similarities and differences: while for both cases we find similar characteristics of the occupational situation associated with working poverty, we find differences in terms of experience. The comparative study of working poverty allows us to better understand its origins and its various consequences, in order to overcome discussions on particular cases and contributing to the creation of a general analytical framework.

Keywords: Labor poverty, Working poor. 


\section{Introducción}

Diversas ${ }^{1}$ transformaciones sociales a escala global han implicado aumento de desigualdad social, mayor incertidumbre e individualización del riesgo (Bauman, 2005a; Beck, 2009; Sennett, 2007). Respecto de la organización del mercado del trabajo tanto en Europa como en Latinoamérica emerge un nuevo panorama social: mayor precarización y merma de las condiciones de trabajo que afecta con mayor fuerza al segmento secundario, retroalimentando y diversificando la estructura de desigualdad (Beck, 2002; Galván, Amarante, \& Mancero, 2016; Sennett, 2007). Mientras en Europa el mercado del trabajo deja a grupos de trabajadores en empleos temporales y con menor protección, imponiendo a los sujetos la necesidad de moverse continuamente entre diversos empleos sin necesariamente contar con las capacidades para ello (Beck, 2002 y 2009; Sennett, 2007), en América Latina la precarización de las condiciones de empleo se da en un escenario altamente desigual (De la Garza, 2016; Julián y Hernández Aracena, 2017).

En este contexto nos encontramos con un grupo de trabajadores, principalmente del segmento secundario, que forman parte de hogares en situación de pobreza. Según la OIT, a nivel mundial el $26 \%$ de los trabajadores no reciben una retribución que les permita satisfacer sus necesidades básicas y de sus familias. De acuerdo a esta medición, se trataría de un fenómeno presente solo en países de ingresos medios y bajos, por lo que no encontraríamos trabajadores en situación de pobreza ni en Norteamérica ni en Europa occidental (OIT, 2019). Sin embargo, si utilizamos indicadores de pobreza de los propios países encontraremos que también se trata de una realidad existente en estas regiones, principalmente después de la crisis económica de 2008.

El trabajo sigue constituyendo una de las principales dimensiones de cohesión social e individualización. El ser humano requiere construir una biografía que interconecte sus experiencias y dé sentido a su existencia, sin embargo, bajo las nuevas condiciones de empleo la satisfacción de esta necesidad se ve en peligro en un mundo que solo permite una vida compuesta de episodios y fragmentos (Sennett, 2007). El capitalismo flexible destruye sistemáticamente las bases para el desarrollo de una personalidad coherente y deja a los individuos con biografías rotas y fragmentadas, sin orientación. La flexibilización característica de nuestra época ha transformado el mundo laboral, generando una profunda contradicción entre el comportamiento que debemos adoptar en el trabajo versus las necesidades de socialización para la reproducción social (Sennett, 2007). La nueva cuestión social es la constante exclusión de vidas humanas que ya no son necesarias para la economía mundial. En el extremo superior de la estructura social encontramos a quienes pueden construir sus vidas a elección, mientras que en el otro extremo encontramos a quienes no se les permite decidir lo que prefieren, debiendo soportar vidas decididas por otros (Bauman, 2000, 2005b).

Las ciencias sociales deben proveer de explicaciones que permitan comprender cuáles son los mecanismos sociales que permiten la persistencia de la pobreza, para de esta manera visibilizar las estructuras que la reproducen. El objetivo de esta contribución es dar cuenta, de manera comparada, de la pobreza laboral en dos contextos diferentes: Chile y España. El análisis de bases de datos de ambos países nos permitirá una exploración de las principales circunstancias sociales que 
influyen en que determinados trabajadores vivencien pobreza laboral y la revisión bibliográfica nos permitirá conocer la experiencia de este grupo de trabajadores, identificando semejanzas y diferencias para ambos contextos.

\section{Perspectiva teórica}

La pobreza no está asociada solamente a situaciones de desempleo, sino que es posible encontrarla en el marco de participación del mercado del trabajo. Paugam (2007) identifica tres formas básicas de la pobreza: por un lado, una pobreza vinculada a una determinada ocupación, un grupo al que denomina de individuos en situación de fragilidad, quienes siguen estando en una categoría social definida por la actividad o trabajo realizado. En segundo lugar, una pobreza lisa y llana, formada por sujetos que, por depender totalmente de la asistencia social, quedan definidos completamente por su condición de asistidos. Finalmente, un tercer grupo formado por individuos que viven situaciones de exclusión social.

Los trabajadores pertenecientes a hogares en situación de pobreza, que corresponden al primer grupo identificado por Paugam, no han perdido totalmente su identidad pero pueden comenzar a vislumbrar situaciones de descalificación social. ${ }^{2}$ La investigación muestra que un número cada vez mayor de asalariados sufren el sentimiento de no tener la más mínima posibilidad de mejorar su suerte (Arnal, Finkel, y Parra, 2013; Calvo y Gómez-Álvarez, 2017; Márquez, 2001; Santamaría, 2009). Si bien es esperable la destrucción de empleo y aumento de la pobreza en periodos de crisis económicas, suponemos el resultado contrario en periodos de crecimiento económico. Sin embargo, luego de la crisis de 2008 el crecimiento económico ha reducido lentamente el desempleo y muchos de los nuevos empleos corresponden a formas atípicas de contratación, contratos a tiempo parcial y temporales. La explicación estaría en que, pese a existir crecimiento económico, este se concentraría en el $1 \%$ superior, disminuyendo la tasa de participación laboral. Para que el crecimiento se traduzca en reducción de la pobreza la creación de empleo debe ir de la mano con mejores condiciones de trabajo, lo que no siempre ocurre (Cruces, Fields, Jaume, y Viollaz, 2017; OECD Employment Outlook 2018, 2018; Piketty, 2014; Stiglitz, 2019).

El concepto de pobreza laboral involucra dos dimensiones; por un lado, la situación de pobreza y, por otro, la participación en el mercado del trabajo. Respecto del concepto de pobreza existen distintos paradigmas para su estudio. Desde el Enfoque de Necesidades Básicas se considera que una persona o un hogar se encuentran en situación de pobreza cuando no pueden satisfacer una serie de necesidades que aseguran la supervivencia. La medición de dicha satisfacción de necesidades puede realizarse de manera directa (con una selección de indicadores que den cuenta de la satisfacción de dichas necesidades) o indirecta (ingresos que permitan satisfacer dichas necesidades), los cuales pueden ser considerados de manera absoluta (independiente de la riqueza de los demás) o relativa (tomando en consideración el nivel de vida del resto de la sociedad) (Feres y Mancero, 2001). Por otro lado, el paradigma de Pobreza Multidimensional se inspira en el enfoque de capacidades de Sen (2000), según el cual la pobreza es privación de capacidades humanas. Desde esta perspectiva se han desarrollado medidas multidimensionales para dar cuenta de la pobreza, las que seleccionan una 
variedad más amplia de indicadores en las dimensiones de educación, salud y calidad de vida (Alkire et al., 2015).

La literatura referida a pobreza laboral se ha remitido a la medición por ingresos a nivel de hogares del enfoque de necesidades. A nivel mundial la OIT utiliza la medición de pobreza monetaria absoluta de $\$ 1.90$ dólares (PPP) per cápita por día (pobreza extrema) o entre $\$ 1.90$ y $\$ 3.20$ dólares (PPP) per cápita por día (pobreza moderada) (OIT, 2019). La UE utiliza una medida relativa de medición de pobreza por ingresos, considerando que un trabajador se encuentra en riesgo de pobreza laboral cuando su ingreso equivalente disponible al año para el hogar es menor que el $60 \%$ del ingreso mediano por unidad de consumo de los hogares a nivel nacional (Eurofound, 2017). En el caso de Chile, la pobreza laboral se ha medido utilizando la medida nacional absoluta de pobreza, aunque también se ha utilizado la medición relativa de ingreso europea para efectos de comparación (Bennett, 2017).

En cuanto a tipo de ingresos que se han tomado en cuenta para distinguir entre hogares en pobreza de los no pobres, también ha habido distintas opciones. Parte de la literatura ha considerado solo los ingresos obtenidos por el trabajo, pero también algunos estudios han considerado todos los ingresos obtenidos por un hogar determinado, tanto por trabajo como por otras fuentes, como transferencias o pensiones. Respecto de la dimensión laboral, se ha debatido respecto de las características que deben cumplir los trabajadores para ser incluidos en el concepto. Parte de la literatura se ha inclinado por incluir solo a quienes se hayan desempeñado por cuenta ajena al menos siete meses en el último año, mientras que otros exigen que la persona haya trabajado todo el último año anterior (Crettaz, 2015; Eurofound, 2017; Halleröd, Ekbrand, y Bengtsson, 2015; Lohmann, 2009). La opción que se adopte, tanto en cuanto al tiempo de trabajo como a si se incluye solo a quienes trabajan como asalariados o también a quienes lo hacen por cuenta propia, va a tener consecuencias en la caracterización y dimensiones del fenómeno.

El carácter multidimensional del concepto de pobreza laboral se ve reflejado en los enfoques teóricos que la describen, identificando factores que la determinan a nivel estructural, a nivel de hogares y de los sujetos. A nivel estructural inciden elementos como el contexto económico, los regímenes de bienestar, el contexto institucional y de segmentación del mercado del trabajo. Los estudios comparados de los sistemas capitalistas contradicen la idea de homogeneidad y convergencia de las economías, planteando que las formas de organización económicas se sitúan en un marco de relaciones sociales más extensas que dan lugar a regímenes económicos específicos a cada contexto (Aguirre y Lo Vuolo, 2013). Así, cada organización económica tendrá diferentes resultados en términos de crecimiento, productividad, generación de empleos e igualdad o desigualdad en la distribución de bienes y servicios, las que influirán en la mayor o menor presencia de pobreza laboral. Las economías de ambos casos de estudio presentan diferencias en términos, por ejemplo, de productividad, diversificación, tasas de crecimiento e innovación, variables que incidirán en la forma y características de la pobreza laboral en cada caso (Fernández, 2011; Julián y Hernández Aracena, 2017; Montoya, 2014; Pérez Ahumada, 2010).

Los regímenes de bienestar aducen a la forma en que se organiza la provisión del bienestar entre Estado, mercado y familia, y en qué medida esta organización 
logra mayores o menores niveles de desmercantilización de las condiciones de vida (Esping-Andersen, 1990). Si encontramos un mayor apoyo por parte del Estado, habrá mayor distribución de recursos monetarios y provisión de servicios a los hogares y, por tanto, existirán menos posibilidades de sufrir pobreza laboral. Al contrario, si los trabajadores deben recurrir principalmente al mercado o al apoyo de las familias para la provisión de bienestar, los hogares serán más dependientes de sus ingresos o de las posibilidades familiares de asumir la provisión de bienestar (De Haan, 2007; Garland, 2015; Heinz y Lund, 2012; Lohmann, 2009). El contexto institucional dice relación con la regulación, prácticas, políticas y convenciones que influyen en las condiciones de contratación laboral, salarios, jornada, negociación colectiva y sindicalización (Betcherman, 2012). Así, regulaciones como salario mínimo, densidad sindical y cobertura de la negociación colectiva determinarán los recursos de poder de los trabajadores y, por tanto, sus posibilidades de incidir en el nivel de salarios y los niveles de desigualdad social (Lohmann, 2009).

Finalmente, las características de segmentación del mercado del trabajo influirán en la pobreza laboral en un determinado contexto. Desde las teorías de segmentación se señala que el mercado del trabajo se encuentra formado por segmentos con características que muestran cierta homogeneidad intrasegmento y cierta distancia respecto de los demás (Alós-Moner, 2007, 2008; Fernández-Huerga, 2010; Gibert, 2011; López-Roldán, Miguélez, Lope, y Coller, 1998). Para identificar y caracterizar los segmentos la literatura ha tenido en cuenta dimensiones como estabilidad/inestabilidad, cualificación/ no cualificación, ingresos, existencia/no existencia de derechos laborales, sector de actividad y tipo y tamaño del establecimiento. Además, se ha considerado variables sociodemográficas de los trabajadores como sexo, edad, educación y origen migrante (López-Roldán y Fachelli, 2017; López-Roldán et al., 1998; López-Roldán, Semenza y Salvia, 2020).

Desde esta perspectiva se identifica la existencia de dos segmentos; por un lado, un segmento primario, superior e inferior, con empleos calificados, protección laboral e ingresos medios y altos y, por otro, un segmento secundario con empleos de baja o muy poca calificación, con contratos temporales, en condiciones precarias y menores salarios (Fernández-Huerga, 2010; López-Roldán y Fachelli, 2017; López-Roldán et al., 1998). Son precisamente las circunstancias que caracterizan a las condiciones de empleo identificadas en el segmento secundario las que más se asocian a encontrarse en pobreza laboral, lo que no significa que ambas categorías sean sinónimas ya que podremos encontrar trabajadores de otros segmentos que formen parte de hogares en situación de pobreza, como también encontraremos trabajadores del segmento secundario que no pertenezcan a hogares en pobreza.

Tanto a nivel de hogares como de los individuos también encontramos elementos que inciden en la pobreza laboral. El tamaño y composición de los hogares incide en la intensidad laboral y, por tanto, en los recursos de los mismos para satisfacer las necesidades de sus integrantes. A nivel individual, elementos como clase de origen, sexo, migración, nivel educativo y empleo determinarán en parte los ingresos disponibles del hogar y, por tanto, las probabilidades de experimentar pobreza laboral (Lohmann y Marx, 2018). Será, por tanto, la 
conjugación final de todos estos elementos lo que determinará la configuración específica de la pobreza laboral en cada contexto.

\section{Contexto para la comparación}

Los estudios comparados en pobreza nos permiten mayor profundidad analítica puesto que posibilitan la comprensión de los contextos desde los cuales esta se produce, superando los debates casuísticos y contribuyendo a la construcción de un marco analítico general susceptible de explicarla. Como la pobreza laboral depende de factores macro, meso y micro sociales, el indagar respecto de todos estos elementos en dos contextos distintos nos permite un mejor entendimiento de su interrelación y un mejor conocimiento de qué aspectos son propios de cada caso y cuáles son comunes a ambos (Tonon, 2011; Piovani y Krawczyk, 2017; Sartori, 1994).

Como casos hemos elegido a Chile y España, representantes de dos regiones diversas. Si bien vivimos en un mundo globalizado, cada caso de estudio tiene sus particularidades históricas, políticas y económicas que deben ser tomadas en cuenta como contexto para la comparación. Mientras trabajadores de países europeos viven situaciones de precariedad impensadas hace un par de años, trabajadores de Latinoamérica deben enfrentar procesos de precarización en un escenario altamente desigual.

\section{Chile en el contexto de América Latina}

En países de ingresos medios y bajos la pobreza laboral no es un problema de que se trabaje poco, sino más bien es consecuencia de la poca retribución por hora trabajada que reciben los trabajadores. Ante la insuficiente demanda laboral y por la inexistencia de seguros de desempleo adecuados, las personas no pueden mantenerse sin desarrollar ningún tipo de actividad y deben recurrir al autoempleo o a actividades de subsistencia. Cuando se está en esta situación se aparece como empleado en las cifras oficiales, pero comúnmente las actividades se realizan en condiciones extremadamente precarias, sin ninguna seguridad de cuánto dinero se recaudará a fin de mes (Fields, 2018). Estas actividades corresponden a trabajo por cuenta propia, pero se les ha denominado sector informal, para diferenciarlo del trabajo por cuenta propia productivo, porque muchas veces se trata de actividades con un escaso margen de retorno (Álvarez, 2005; Prez, 2014; Saraví, 2006). Tambin es posible encontrar pobreza laboral en el marco de empleo por cuenta ajena en empresas de alto retorno económico o en empresas que prestan servicios a dichas empresas. En este caso, por las condiciones institucionales de escasa protección del trabajo y los bajos niveles de sindicalización y negociación colectiva, los trabajadores cuentan con menor poder de negociación, lo que se traduce en bajos salarios (Julián y Hernández Aracena, 2017).

Para el caso de América Latina se ha estudiado la pobreza laboral comparativamente en cinco países, identificando dos grupos: por un lado, Argentina, Brasil y Costa Rica, con una incidencia aproximada del $11 \%$ y, por otro, Perú y Ecuador, donde alcanza aproximadamente el $25 \%$. Conjuntamente 
con las condiciones estructurales ya mencionadas, a nivel de hogares la mayor presencia de niños incide en mayor pobreza laboral. Además de las mayores necesidades de los hogares con hijos, las obligaciones de cuidado de los mismos imponen importantes restricciones para la participación de la mujer en el mercado del trabajo, en un escenario de ausencia de políticas públicas de cuidado. A nivel individual, a mayor nivel educativo y mayor edad existen menores posibilidades de pobreza laboral, mientras que la misma afecta de menor manera a mujeres que hombres (Lohmann y Marx, 2018).

\section{España como parte de Europa}

Serían fenómenos como la globalización, la desindustrialización y el cambio tecnológico las principales variables que habrían perjudicado a los trabajadores de baja calificación en los países de ingresos altos (Crettaz, 2013). En Europa la pobreza laboral afecta en mayor medida a trabajadores cuyo salario es el único sostn de su familia, ya sea familias monoparentales o formadas por parejas, pero con un solo perceptor de ingresos. Es decir, el percibir un salario bajo no es sinónimo de pobreza laboral, sino que depende tambin de elementos a nivel de hogares, como el tamao y número de adultos con trabajo, y de elementos de contexto, como el régimende bienestar (Filandri y Struffolino, 2019; Goerne, 2010; Marx y Nolan, 2013).

El tener un empleo de bajo salario y bajo nivel educativo aumenta posibilidades de estar en pobreza laboral. En el caso del sexo, las mujeres ayudan a la familia a escapar de la pobreza laboral cuando constituyen un segundo ingreso, pero si son la jefa de hogar aumenta la posibilidad de ser una trabajadora en situación de pobreza. Otros factores relevantes a nivel individual que incrementan las posibilidades de encontrarse en pobreza laboral son la condición de migrante, el tipo de contrato, trabajar una jornada parcial, tener un contrato temporal y ser auto empleado (Eurofound, 2017). A nivel del hogar, la composición del mismo es uno de los principales factores. El mayor riesgo de encontrarse en pobreza laboral se da en aquellos trabajadores pertenecientes a hogares con niños, principalmente hogares monoparentales o con dos padres, pero con tres hijos o más. La variable realmente determinante es la cantidad de adultos trabajando y la intensidad laboral del hogar completo, lo que determina la ratio entre el número de trabajadores y el número de dependientes (Eurofound, 2017).

A nivel institucional, los niveles de desfamiliarización y desmercantilización de cada régimen de bienestar inciden en las tasas de pobreza laboral en cada país. Así, los países nórdicos, caracterizados por amplios niveles de desmercantilización y desfamiliarización, tienen los niveles más bajos de pobreza laboral en la UE. Por el contrario, en regímenes liberales donde las políticas sociales están concentradas en garantizar solo un mínimo para la población más vulnerable, se encuentran los niveles más bajos de desmercantilización y desfamiliarización de toda la UE, con resultados disímiles en términos de pobreza laboral. Los países pertenecientes al modelo continental, caracterizados por bajos niveles de desfamiliarización pero niveles considerables de desmercantilización, obtienen resultados medios en términos de pobreza laboral, encontrándose comparativamente en una situación intermedia entre los dos regímenes anteriores. Finalmente, los países del sur de Europa tienen similares niveles de desmercantilización respecto del modelo 
continental pero menores niveles de desfamiliarización, resultando en mayores niveles de pobreza laboral (Eurofound, 2017).

\section{Pobreza laboral en Chile y España}

Los contextos de cada uno de los casos van a incidir en la mayor o menor presencia de pobreza laboral. Respecto de España, se trata de una economía de capitalismo central de desarrollo tardío, de ingresos altos, con elementos del modelo liberal y abierta al intercambio comercial en el marco de la UE (Berzosa, 2008; Fernández, 2011; Recio, 2010), mientras que la economía chilena pertenece a la semiperiferia, con ingresos medios, calificada como economía de mercado jerárquica, con amplias diferencias de productividad entre sus distintos sectores, alta concentración económica y altos niveles de desigualdad (Aguirre y Lo Vuolo, 2013; Ffrench-Davis, 2010).

En cuanto a los regímenes de bienestar, el modelo español presenta un nivel medio de desmercantilización, persistencia de brechas de desigualdad y dependencia en la familia para la provisión de bienestar (Banyuls, Miguélez, Recio, Cano, y Lorente, 2009). Sin embargo, recientemente se han implementado medidas para hacer frente a la pobreza y la exclusión social, como las Rentas mínimas de inserción y el Ingreso mínimo vital, lo que viene a mejorar la red de protección social (Ministerio de Derechos Sociales y Agenda 2030, 2020; Ministerio de Sanidad, 2020). Por su parte, el modelo chileno ha sido calificado como "estatal productivista", puesto que enfatiza la formación de capital humano, versus un menor énfasis en la desmercantilización de las condiciones de vida. El Estado adopta un carácter subsidiario, por tanto promueve la participación en el mercado y depende ampliamente de la familia para la provisión de bienestar (Martínez, 2007; MIDEPLAN, 1991).

En cuanto al contexto institucional, en España las reformas laborales han introducido la flexibilidad en la relación laboral, fortalecido el poder de la empresa, debilitado el rol de los sindicatos y favorecido las formas atípicas de contratación (Alós-Moner, Beneyto, y Jódar, 2017). En el caso chileno, la reforma de 1979 flexibilizó significativamente la legislación, permitiendo el despido no justificado, restringiendo los derechos sindicales, delimitando la negociación colectiva a nivel de empresa, permitiendo el reemplazo de trabajadores en huelga, la negociación con grupos de trabajadores, haciendo voluntaria la afiliación sindical y recortando el monto de la indemnización por despido (Narbona, Páez, y Tonelli, 2011).

Finalmente, respecto de la estructura de segmentación del mercado del trabajo, en España encontramos un segmento primario superior del $30 \%$ del mercado laboral, formado por el empleo de mejor calidad, sector en el cual predominan las mujeres y las personas de mayor edad. El segmento primario inferior representa el $52 \%$ del mercado, en el cual la gran mayoría cuenta con contrato indefinido, niveles medios de salarios, en sectores como industria, construcción, comercio y transporte (López-Roldán et al., 2020). El segmento secundario representa el $18 \%$ de los trabajadores, formado principalmente por trabajadores no calificados con contratos temporales o empleados a tiempo parcial y con escasa antigüedad laboral (López-Roldán et al., 2020). Por su parte, para Chile se ha identificado 
un segmento primario superior del $24 \%$, un segmento primario inferior del $47 \%$ y un segmento secundario del 29\% (López-Roldán et al., 2020).

En cuanto a las características de la pobreza laboral, se presentan diferencias en ambos casos. En Chile la presencia de trabajadores en situación de pobreza es un elemento estructural del modelo de capitalismo. Si bien las cifras de pobreza han disminuido de manera consistente en el tiempo, fruto de transferencias monetarias focalizadas, los niveles de salario no han mejorado (Gammage, Alburquerque y Durán, 2014). A nivel estructural la pobreza laboral se explicaría por variables como la deficiente oferta laboral, que obliga a las personas a ejercer actividades de subsistencia, la deficiente regulación del mercado del trabajo, los bajos salarios y un frágil régimen de bienestar (Lohmann y Marx, 2018). A nivel de hogares e individuos, la mayor incidencia de pobreza laboral se da en trabajadores con bajos niveles educativos, pertenecientes a familias extensas con baja tasa de empleo, en empleos de bajos salarios o que ejercen actividades informales o a tiempo parcial, en empresas pequeñas o de sectores de baja tecnología como agricultura y comercio (Bennett, 2017).

En España el crecimiento económico y las políticas sociales del Estado de Bienestar habían logrado disociar el trabajo de la pobreza. Sin embargo, especialmente después de la crisis de 2008, ha aumentado la incidencia de pobreza entre los trabajadores, la cual encuentra en la calidad del empleo su principal variable explicativa (Banyuls y Recio, 2017; Lope, 2015). España tiene uno de los más altos niveles de pobreza laboral de Europa occidental, debido a la ausencia de políticas que logren mayores niveles de desfamiliarización y por falta de protección en segmentos del mercado laboral ocupado típicamente por jóvenes, mujeres y trabajadores con baja calificación (Banyuls y Recio, 2017; Eurofound, 2017; García Espejo y Gutiérrez, 2011; Tejero Pérez, 2018). Sin embargo, también encontramos trabajadores hombres a tiempo completo en situación de pobreza laboral, asociado al hecho de que ser los únicos proveedores en hogares de gran tamaño (Halleröd et al., 2015; Tejero Pérez, 2018).

Para dar cuenta de las características de la pobreza laboral en ambos contextos hemos analizado las bases de datos de ambos de países; para el caso de Chile utilizamos la CASEN 2017 y para España la ECV 2018. Hemos definido pobreza laboral como la situación de los trabajadores que pertenecen a hogares en pobreza. Para la determinación de los hogares en pobreza hemos tomamos como línea de pobreza la medición europea, es decir, el $60 \%$ de la mediana de ingresos por unidad de consumo por hogar y hemos considerado los ingresos totales del hogar. Respecto de la identificación de los trabajadores hemos considerado como tal a quien haya declarado trabajar el año anterior, independientemente del tiempo que lo haya realizado e independientemente de si trabajó por cuenta ajena o por cuenta propia.

Si bien los datos arrojan una menor cantidad de hogares en situación de pobreza para el caso de Chile, ello no significa que las condiciones socioeconómicas sean efectivamente mejores, sino que más bien es consecuencia de la forma de medición ocupada. Lo importante es conocer las semejanzas y diferencias entre los trabajadores que pertenecen a hogares en pobreza versus quienes no se encuentran en esta condición. Para ambos casos, el ingreso per cápita de los hogares en situación de pobreza representa aproximadamente un tercio del ingreso per cápita del resto de los hogares, el tamaño de los hogares 
en pobreza es leventemente superior y el porcentaje de integrantes ocupados es significativamente menor. De esta manera, a nivel de hogares encontramos los elementos que la literatura ha considerado relevantes en la pobreza laboral: son hogares con menores ingresos producto, en parte, de que tienen un menor porcentaje de personas ocupadas, es decir, menor intensidad laboral y, por tanto, mayor porcentaje de personas dependientes.

Tabla 1

\begin{tabular}{|c|c|c|c|c|}
\hline \multirow{2}{*}{} & \multicolumn{2}{|c|}{ CHILE } & \multicolumn{2}{c|}{ ESPAÑA } \\
\cline { 2 - 5 } & POBRE & $\begin{array}{c}\text { NO } \\
\text { POBRE }\end{array}$ & POBRE & $\begin{array}{c}\text { NO } \\
\text { POBRE }\end{array}$ \\
\hline HOGARES & $17,6 \%$ & $82,4 \%$ & $20,5 \%$ & $79,5 \%$ \\
\hline $\begin{array}{c}\text { INGRESO DEL HOGAR } \\
\text { PER CÁPITA } \\
\text { MENSUAL PROMEDIO }\end{array}$ & $156 €$ & $538 €$ & $307 €$ & $1076 €$ \\
\hline $\begin{array}{c}\text { MIEMBROS DEL } \\
\text { HOGAR }\end{array}$ & 3,4 & 3 & 2,6 & 2,5 \\
\hline $\begin{array}{c}\text { INTEGRANTES } \\
\text { OCUPADOS }\end{array}$ & $37,6 \%$ & $58,7 \%$ & $32,6 \%$ & $52,9 \%$ \\
\hline
\end{tabular}

Fuente: elaboración propia sobre la base de CASEN 2017 y ECV 2018

Para analizar de las características de los trabajadores se utilizaron las variables identificadas por la literatura como significativas a nivel individual.

Tabla 2a

\begin{tabular}{|c|c|c|}
\hline VARIABLES & DESCRIPCIÓN & CATEGORIAS \\
\hline SEXO & Sexo del encuestado & Mujer \\
& & Varón \\
\hline PAIS DE & País de nacimiento del & Nacional \\
NACIMIENTO & encuestado & Extranjero \\
\hline CALIFICACIÓN & Nivel de calificación & Semi calificado \\
& & No calificado \\
\hline DEPENDENCIA & Situación de & Patrón \\
& dependencia laboral & Asalariado \\
& & Cuenta propia \\
& & Familiar no remunerado \\
\hline
\end{tabular}


Tabla 2b

\begin{tabular}{|c|c|c|}
\hline VARIABLES & DESCRIPCION & CATEGORIAS \\
\hline SECTOR ACTIVIDAD & $\begin{array}{l}\text { Rama de actividad } \\
\text { económica }\end{array}$ & $\begin{array}{c}\text { Extractivo } \\
\text { Manufactura } \\
\text { Servicios } \\
\text { Administración pública }\end{array}$ \\
\hline $\begin{array}{c}\text { TAMAÑO } \\
\text { ESTABLECIMIENTO }\end{array}$ & $\begin{array}{l}\text { Tamaño de empresa según } \\
\text { número de trabajadores }\end{array}$ & $\begin{array}{l}\text { Unipersonal } \\
\text { Pequeña } \\
\text { Mediana } \\
\text { Grande }\end{array}$ \\
\hline TIPO CONTRATO & $\begin{array}{c}\text { Clasificación de contrato en } \\
\text { base a duración en el } \\
\text { tiempo }\end{array}$ & $\begin{array}{l}\text { Indefinido } \\
\text { A plazo fijo }\end{array}$ \\
\hline JORNADA LABORAL & $\begin{array}{c}\text { Clasificación de jornada } \\
\text { laboral en base a horas } \\
\text { trabajadas semanalmente } \\
\text { (jornada completa son } 40 \\
\text { horas en España y } 45 \\
\text { horas en Chile) }\end{array}$ & $\begin{array}{l}\text { Completa } \\
\text { Parcial }\end{array}$ \\
\hline
\end{tabular}

Respecto de la cantidad de trabajadores en hogares en situación de pobreza encontramos cifras similares para ambos casos. Las variables de sexo, edad y horas trabajadas semanalmente no presentan mayores diferencias entre ambos grupos en ninguno de los dos países. En cambio, en ambos contextos los trabajadores pertenecientes a hogares en situación de pobreza tienen menos años de escolaridad e ingresos laborales significativamente menores. En cuanto al país de nacimiento, no se presentan diferencias para el caso de Chile mientras que para España los trabajadores migrantes se ven más representados en el grupo de trabajadores pertenecientes a hogares en pobreza.

En cuanto a la calificación profesional, en ambos casos los trabajadores pertenecientes a hogares en pobreza tienen más representación entre los trabajadores no calificados y menor representación el grupo de trabajadores calificados. En cuanto a la dependencia los trabajadores de hogares en situación de pobreza tienen menor representación entre los asalariados y mayor representación entre los trabajadores por cuenta propia. En cuanto al sector de actividad los trabajadores de hogares en pobreza tienen mayor presencia en el sector extractivo y menor en la administración pública en ambos casos. Finalmente, los trabajadores de hogares en pobreza trabajan en mayor medida en establecimientos de menor tamaño y tienen mayor presencia en la contratación a plazo fijo y en jornada parcial, en comparación con el resto de los trabajadores en ambos países. 
Tabla 3a

\begin{tabular}{|c|c|c|c|c|c|}
\hline \multirow{2}{*}{} & \multicolumn{2}{c|}{ CHILE } & \multicolumn{2}{c|}{ ESPAN̈A } \\
\cline { 2 - 6 } & POBRE & NO POBRE & POBRE & NO POBRE \\
\hline \multirow{2}{*}{ TRABAJADORES } & $12,6 \%$ & $87,4 \%$ & $13,8 \%$ & $86,2 \%$ \\
\hline \multirow{2}{*}{ SEXO MUJER } & $41,6 \%$ & $43,8 \%$ & $43,5 \%$ & $45,6 \%$ \\
\cline { 2 - 6 } & HOMBRE & $58,4 \%$ & $56,2 \%$ & $56,5 \%$ & $54,4 \%$ \\
\hline \multicolumn{2}{|c|}{ EDAD } & 43 & 43 & 44 & 43 \\
\hline \multirow{2}{*}{$\begin{array}{c}\text { AN PAIS DE DE ESCOLARIDAD } \\
\text { NACIMIENTO }\end{array}$} & NACIONAL & $96,5 \%$ & $95,4 \%$ & $63 \%$ & $88,1 \%$ \\
\cline { 2 - 6 } & EXTRANJERO & $3,5 \%$ & $4,6 \%$ & $37 \%$ & $11,9 \%$ \\
\hline
\end{tabular}

Fuente: elaboración propia sobre la base de CASEN 2017 y ECV 2018

Tabla 3b

\begin{tabular}{|c|c|c|c|c|c|}
\hline & & \multicolumn{2}{|c|}{ CHILE } & \multicolumn{2}{|c|}{ ESPAN̈A } \\
\hline & & POBRE & NO POBRE & POBRE & NO POBRE \\
\hline \multicolumn{2}{|c|}{$\begin{array}{l}\text { PROMEDIO HORAS TRABAJADAS } \\
\text { SEMANALMENTE }\end{array}$} & 39,8 & 42,9 & 37,7 & 38,8 \\
\hline \multicolumn{2}{|c|}{ INGRESO LABORAL MENSUAL PROMEDIO } & $309 €$ & $668 €$ & $405 €$ & $1379 €$ \\
\hline \multirow{3}{*}{ CALIFICACION } & CALIFICADO & $12 \%$ & $30,1 \%$ & $14,2 \%$ & $36,7 \%$ \\
\hline & SEMI CALIFICADO & $55,2 \%$ & $49,5 \%$ & $58,3 \%$ & $52 \%$ \\
\hline & NO CALIFICADO & $32 ; 8 \%$ & $20,4 \%$ & $27,6 \%$ & $11,3 \%$ \\
\hline \multirow{4}{*}{ DEPENDENCIA } & PATRON & $1,4 \%$ & $2,4 \%$ & $7,7 \%$ & $4,4 \%$ \\
\hline & ASALARIADOS & $66,1 \%$ & $77,2 \%$ & $73,6 \%$ & $86 \%$ \\
\hline & CUENTA PROPIA & $31,7 \%$ & $20,1 \%$ & $18,6 \%$ & $9,5 \%$ \\
\hline & FNR & $0,8 \%$ & $0,4 \%$ & $0,2 \%$ & $0,1 \%$ \\
\hline
\end{tabular}

Fuente: elaboración propia sobre la base de CASEN 2017 y ECV 2018 
Tabla 3c

\begin{tabular}{|c|c|c|c|c|c|}
\hline & & \multicolumn{2}{|c|}{ CHILE } & \multicolumn{2}{|c|}{ ESPAÑA } \\
\hline & & POBRE & NO POBRE & POBRE & NO POBRE \\
\hline \multirow{4}{*}{$\begin{array}{c}\text { SECTOR } \\
\text { ACTIVIDAD }\end{array}$} & EXTRACTIVO & $18,3 \%$ & $9,9 \%$ & $11,5 \%$ & $3,9 \%$ \\
\hline & MANUFACTURA & $21 \%$ & $18,9 \%$ & $18,2 \%$ & $19,5 \%$ \\
\hline & SERVICIOS & $46,9 \%$ & $47,8 \%$ & $59,8 \%$ & $50,2 \%$ \\
\hline & $\begin{array}{l}\text { ADMINISTRACIÓN } \\
\text { PÜBLICA }\end{array}$ & $13,8 \%$ & $23,4 \%$ & $9,9 \%$ & $26 \%$ \\
\hline \multirow{4}{*}{$\begin{array}{c}\text { TAMAN̈O } \\
\text { ESTABLECIMIENTO }\end{array}$} & UNIPERSONAL & $33,2 \%$ & $19,7 \%$ & $23,9 \%$ & $9,9 \%$ \\
\hline & PEQUEÑA & $23,9 \%$ & $20,5 \%$ & $39,9 \%$ & $25,6 \%$ \\
\hline & MEDIANA & $14,9 \%$ & $17,9 \%$ & $21,2 \%$ & $28,5 \%$ \\
\hline & GRANDE & $27,9 \%$ & $41,9 \%$ & $15,1 \%$ & $36,1 \%$ \\
\hline \multirow{2}{*}{ TIPO CONTRATO } & INDEFINIDO & $58,6 \%$ & $73,9 \%$ & $48,3 \%$ & $77,5 \%$ \\
\hline & PLAZO FIJO & $41,4 \%$ & $26,1 \%$ & $51,7 \%$ & $22,5 \%$ \\
\hline \multirow{2}{*}{ JORNADA } & COMPLETA & $85,2 \%$ & $90,2 \%$ & $77,2 \%$ & $89,4 \%$ \\
\hline & PARCIAL & $14,8 \%$ & $9,8 \%$ & $22,8 \%$ & $10,6 \%$ \\
\hline
\end{tabular}

Fuente: elaboración propia sobre la base de CASEN 2017 y ECV 2018

En cuanto a la vivencia de la pobreza laboral, existen para ambos casos diversos estudios que han indagado en la experiencia de los trabajadores en situación de pobreza. El PNUD ha identificado para Chile distintos perfiles. Un primer grupo está integrado por trabajadores que se desempeñan principalmente en labores de aseo y ornato, donde hay una alta presencia de mujeres jefas de hogar de baja escolaridad, sin estudios superiores ni capacitaciones formales. Las condiciones laborales son precarias, muchas veces no hay contrato o tienen un contrato a plazo fijo con una empresa subcontratista, las jornadas suelen ser extensas y los ingresos se ubican en torno al ingreso mínimo. En un segundo grupo encontramos trabajadores que logran desarrollar un camino de cierto avance durante su historia laboral, comenzando en actividades no calificadas y luego desarrollándose en la especialización de un oficio. En este caso se trata principalmente de hombres con baja escolaridad que se capacitan en el mismo trabajo (Cárdenas et al., 2014).

Las experiencias laborales de los trabajadores en situación de pobreza dan cuenta de inserciones tempranas al mercado del trabajo, muchas veces abandonando la educación por las necesidades económicas apremiantes de las familias. El mundo del trabajo aparece fuertemente vinculado a lazos familiares y sociales, y son estas redes las que facilitan el acceso, pero por la limitación de los contactos se trata en su mayoría de oportunidades precarias. Tanto hombres como mujeres trabajan mientras están solteros, pero una vez formada la familia son casi exclusivamente los hombres quienes continúan participando en el 
mercado el trabajo, asumiendo la función de proveedor, mientras la mujer asume el cuidado de los niños y el hogar común, alternando con pequeños trabajos independientes de manera esporádica. Los trabajos de quienes se encuentran en situación de pobreza por lo general se desarrollan en rubros con poca fiscalización de derechos laborales, o se trata de trabajo por cuenta propia muchas veces con escaso margen de ganancia. En términos salariales, en los casos en que hay un contrato se tiene certeza del monto del emolumento; en cambio, cuando se trata de una actividad por cuenta propia no existe ninguna seguridad de cuánto se obtendrá a fin de mes (Espinoza y Canteros, 2001).

En las trayectorias en pobreza laboral el proceso de integración social depende de la consistencia del proyecto de familia y de contar con un ingreso económico permanente, por lo cual la posibilidad de escapar de la pobreza es incierta y muchas veces es solo una apuesta intergeneracional. Es muy difícil reponerse de un quiebre en la trayectoria, muchas veces es necesario aferrarse a lo seguro o se debe recurrir a un sinnúmero de contactos familiares o de redes más extensas para no caer en el desempleo. Sin embargo, muchas veces el término de una relación laboral marca el fin de la trayectoria, siendo muy difícil volver a incorporarse al mercado del trabajo (Márquez, 2001).

Las vivencias de trabajadores pertenecientes a hogares en pobreza dan cuenta de experiencias similares producto del espacio social desde el cual son construidas, desde la pobreza y la exclusión social. Sin embargo, pese a este piso común, existe posibilidad de cambios a lo largo del tiempo, existen puntos de inflexión y lógicas de acción que permiten construir una historia diferente. Son los sujetos que logran movilizar una mayor variedad de lógicas de acción los que logran cierta movilidad. Sin embargo, en general, las trayectorias ocupacionales de los más pobres señalan que los trabajos a los que ellos acceden son de tal precariedad e inestabilidad, que la posibilidad de hacer de ellos un recurso para levantarse como sujetos y concretar un proyecto de movilidad social, es a menudo inimaginable (Márquez, 2001, p. 224).

En España también se ha estudiado a los trabajadores en situación de vulnerabilidad, en el contexto poscrisis económica de 2008, identificándose distintos perfiles laborales. Por un lado, encontramos a trabajadores con una larga trayectoria laboral, pero que por la crisis se han visto desestabilizados. Se trata de personas de entre 35 y 45 , quienes si bien se encontraban en trabajos precarios habían logrado mantenerse trabajando de manera continua. Un segundo grupo se compone de personas que no llevaban tanto tiempo en el mercado laboral al momento de la crisis y por tanto no habían alcanzado a estabilizarse ni a adquirir mucha experiencia laboral. Se trata principalmente de personas jóvenes o migrantes de mayor edad (Arnal et al., 2013).

Ambos grupos de trabajadores manifiestan sentirse sobrepasados por la crisis económica, sin los recursos necesarios para reinsertarse, situación que les produce inseguridad y temor. Los relatos de los trabajadores en los márgenes del mercado laboral hablan de las presiones, el malestar, la inseguridad, la inestabilidad y el descontento que implica el tener que "buscarse la vida". Destaca el sentimiento de inseguridad, que se manifiesta en una sensación de indeterminación, de carecer de control, algo que sobrepasa, provocando inseguridades que se traspasan a la biografía. Se trata de una constante amenaza de ser excluido del mercado 
laboral de manera permanente o de mantenerse en las actuales condiciones de precariedad para toda la vida (Arnal et al., 2013).

En la dimensión material, la precariedad salarial se manifiesta en la vivencia de carencias, en la imposibilidad de acceder a la satisfacción de ciertas necesidades y de vivir el nivel de vida que vive la mayoría de la sociedad. Esta situación obliga a la necesidad de realizar todo tipo de artimañas para llegar a fin de mes y recurrir a la lógica del crédito y endeudamiento (Santamaría, 2009). Los trabajadores en empleos precarios relatan abuso de autoridad, demandas irracionales, explotación, sentimiento de injusticia. También afecta la dimensión de construcción de la subjetividad, puesto que características como la temporalidad y la flexibilidad impiden planificar a largo plazo o construir una identidad grupal o individual que dé sentido a la vida, afectado el sentido de autovalía y autoestima (Amable, 2008).

\section{Conclusiones}

Las transformaciones sociales de las últimas décadas han afectado las diversas regiones del mundo, expresándose en cada contexto con especificidades particulares. En el mundo del trabajo estas transformaciones han implicado, principalmente para parte de los trabajadores del segmento secundario, mayor incertidumbre, precariedad y desestabilización de las condiciones laborales. En este contexto hemos abordado de manera comparada la situación de los trabajadores pertenecientes a hogares en pobreza.

En los casos de análisis, por tratarse de contextos diversos, existen mecanismos específicos para cada caso. El contexto del caso chileno se caracteriza por una economía con alta desigualdad, en que conviven sectores productivos con actividades de subsistencia, con un deficiente régimen de políticas sociales y escaso poder de negociación de los trabajadores, lo que genera un mercado laboral marcado por los bajos salarios. Por su parte, en el caso español se trata de un contexto económico más equitativo y homogéneo en términos de productividad, con un régimen de políticas sociales más robusto y mayor poder de negociación de los trabajadores.

Para el caso chileno los principales mecanismos dicen relación, por un lado, con la deficiente demanda laboral y, por tanto, la necesidad de muchas personas de ejercer actividades de subsistencia y, por otro, los bajos salarios del sector formal producto de los escasos recursos de poder de los trabajadores. Para el caso español, en cambio, la pobreza laboral se relaciona con la presencia de niños en los hogares y, ante falta de políticas sociales que garanticen mayores niveles de desfamiliarización, la necesidad de que un adulto asuma las labores de cuidado, generando la presencia de solo un perceptor de ingresos en el hogar.

Sin embargo, y pese a tratarse de contextos diferentes, en ambos casos podemos ver que en los hogares en situación de pobreza el porcentaje de miembros ocupados es significativamente menor y son las características de las condiciones laborales las que presentan mayores diferencias si comparamos los trabajadores de ambos tipos de hogares. Los trabajadores pertenecientes a hogares en situación de pobreza tienen mayor representación entre quienes se desempeñan en jornada parcial, con un contrato temporal, asalariados en una pequeña empresa o por cuenta propia y en puestos de menor calificación. 
En cuanto a la experiencia, en ambos contextos la pobreza laboral dice relación con una situación de fragilidad e inseguridad. Sin embargo, es en el caso chileno donde se perciben trayectorias laborales más marcadas por la privación material. En el contexto de un débil régimen de protección social, con baja calidad de los servicios públicos, las posiciones más desaventajadas en el mercado del trabajo degradan considerablemente las condiciones de vida. A ello debemos sumar la dimensión del tiempo. Mientras en Chile la pobreza laboral es una realidad transmitida de generación en generación, produciendo una acumulación de desventajas, en España se trata de un fenómeno que había logrado mermar y solo resurge tras la crisis de 2008, produciendo la desestabilización de quienes alguna vez lograron cierta estabilidad. Ante la precariedad e incertidumbre del mercado del trabajo, elementos contextuales como el régimen bienestar logran mejores niveles de desmercantilización, mientras que regímenes subsidiarios dejan a los trabajadores y sus familias obligados a asumir el riesgo individualmente.

\section{Referencias}

Aguirre, J., y Lo Vuolo, R. (2013). Variedades de capitalismo. Una aproximación al estudio comparado del capitalismo y sus aplicaciones para América Latina. Documentos de Trabajo CIEPP. https://doi.org/10.3390/w9040285

Alkire, S., Foster, J., Seth, S., Santos, M. E., Roche, J. M., y Ballon, P. (2015). Multidimensional poverty measurement and analysis. Oxford University Press. ht tps://doi.org/10.1017/CBO9781107415324.004

Alós-Moner, R. de. (2007). Mercat, classe i persona en les relacions laborals. Entre la individualitat i l'accio col.lectiva. Universidad Autónoma de Barcelona, España.

Alós-Moner, R. de. (2008). Segmentación de los mercados de trabajo y relaciones laborales. El sindicalismo ante la acción colectiva. Cuadernos de Relaciones Laborales, 26(1), 123-148.

Alós-Moner, R. de, Beneyto, P., y Jódar, P. (2017). Reforma laboral y desregulación del mercado de trabajo. Anuario IET de Trabajo y Relaciones Laborales, 4, 73-86. h ttps://doi.org/10.5565/rev/aiet.55

Álvarez, S. (Ed.). (2005). Trabajo y producción de la pobreza en Latinoamérica y el Caribe\#: estructuras, discursos y actores. Buenos Aires: CLACSO.

Amable, M. (2008). La precariedad laboral y su impacto en la salud. Un estudio en trabajadores asalariados de España. Universidad Pompeu Fabra, España.

Arnal, M., Finkel, L., y Parra, P. (2013). Crisis, desempleo y pobreza: análisis de trayectorias de vida y estrategias. Cuadernos de Relaciones Laborales, 31(2), 281311. https://doi.org/10.5209/rev_crla.2013.v31.n2.43221

Banyuls, J., Miguélez, F., Recio, A., Cano, E., y Lorente, R. (2009). The transformation of the employment system in Spain: Towards a Mediterranean neoliberalism? In G. Bosch, S. Lehndorff, \& J. Rubery (Eds.), European employment models in flux: a comparison of institutional change in nine european countries (pp. 247-269). Palgrave Macmillan UK. https://doi.org/10.1057/9780230237001

Banyuls, J., y Recio, A. (2017). Pobreza laboral en España: causas y alternativas políticas. Anuario IET de Trabajo y Relaciones Laborales, 4(4), 135-149. https://doi.org/ $10.5565 /$ rev/aiet.59

Bauman, Z. (2000). Trabajo, consumismo y nuevos pobres. Barcelona: Editorial Gedisa.

Bauman, Z. (2005a). La globalización. Consecuencias humanas. Polity Press. Cambridge. 
Bauman, Z. (2005b).Vidas desperdiciadas La modernidad y susparias. Barcelona: Paidós.

Beck, U. (2002). Individualization. London: SAGE Publications.

Beck, U. (2009). World at Risk. Cambridge: Polity Press.

Bennett, F. (2017). Size and profile of the working poor in Chile (No. 10). Turin.

Berzosa, C. (2008). Los modelos de desarrollo tardíos. Revista de Economía Mundial, $18,51-63$.

Betcherman, G. (2012). Labor Market Institutions. A Review of the Literature.

Calvo, F., y Gómez-Álvarez, R. (2017). Trabajadores pobres y pobreza en el trabajo. Murcia: Ediciones Laborum.

Cárdenas, A., Undurraga, R., Altschwager, C., Leiva, M., Badilla, M., y Prado, X. (2014). Informe Final: "Estudio trayectorias laborales de la clase media-baja en Chile".

Crettaz, E. (2013). A state-of-the-art review of working poverty in advanced economies: Theoretical models, measurement issues and risk groups. Journal of European Social Policy, 23(4), 347-362. https://doi.org/10.1177/0958928713507470

Crettaz, E. (2015). Poverty and material deprivation among European workers in times of crisis. International Journal of Social Welfare, 24(4), 312-323. https://doi.org $/ 10.1111 /$ ijsw. 12132

Cruces, G., Fields, G. S., Jaume, D., \& Viollaz, M. (2017). Growth, Employment, and Poverty in Latin America (Vol. 1). Oxford University Press. https://doi.org/10.1 093/oso/9780198801085.001.0001

De Haan, A. (2007). Reclaiming social policy: Globalization, social exclusion and new poverty reduction strategies. https://doi.org/10.1057/9780230592285

De la Garza, E. (2016). Los estudios laborales en America Latina. Origenes, desarrollo y perspectivas. (E. De la Garza, Ed.). Barcelona: Anthropos Editorial.

Esping-Andersen, G. (1990). The Three Worlds of Welfare Capitalism. Cambridge: Polity Press, Ed.

Espinoza, V., y Canteros, E. (2001). Contactos sociales y carreras laborales en hogares chilenos de escasos recursos. En Proposiciones: El trabajo en Chile: Aportes desde la investigación. Santiago: Ediciones Sur

Eurofound. (2017). In-work poverty in the EU. Eurostat Methodologies and Working Papers. https://doi.org/10.2785/54568

Feres, J. C., y Mancero, X. (2001). Enfoques para la medición de la pobreza. Breve revisión de la literatura. Santiago: CEPAL

Fernández-Huerga, E. (2010). La teoría de la segmentación del mercado de trabajo: enfoques, situación actual y perspectivas de futuro. Investigación Económica, $\operatorname{LXIX(273),115-150.}$

Fernández, D. (2011). El modelo económico español: una economía abierta y descentralizada. Estudios Internacionales, 43(168), 199-249. https://doi.org/10. $2307 / 41970558$

Ffrench-Davis, R. (2010). Economic Reforms in Chile. London: Palgrave Macmillan UK. https://doi.org/10.1057/9780230289659

Fields, G. S. (2018). Aid, Growth, and Jobs: A Five-Part Policy Framework. In Employment and Development: How Work Can Lead From and Into Poverty (Vol. 13, pp. 25-32). Oxford University Press. https://doi.org/10.1093/oso/9780198 815501.003 .0003

Filandri, M., \& Struffolino, E. (2019). Individual and household in-work poverty in Europe: understanding the role of labor market characteristics. European Societies, 21(1), 130-157. https://doi.org/10.1080/14616696.2018.1536800 
Galván, M., Amarante, V., \& Mancero, X. (2016). Inequality in Latin America: A global measurement. CEPAL Review, 2016(118), 25-44. https://doi.org/10.18356/a7 337ed5-en

Gammage, S., Alburquerque, T., \& Durán, G. (2014). Poverty, inequality and employment in Chile. ILO Working Papers, 46.

García Espejo, I., \& Gutiérrez, R. (2011). Spain: Persisting Inequalities in a Growing Employment Context. In N. Fraser, R. Gutierrez, \& R. Peña-Casas (Eds.), Working Poverty in Europe: A Comparative Approach (pp. 133-154). Palgrave Macmillan.

Garland, D. (2015). The welfare state: A fundamental dimension of modern government. Archives Europeennes de Sociologie (Vol. 55). https://doi.org/10.10 $17 /$ S0003975614000162

Gibert, F. (2011). El mercat de treball metall-mecànic a Catalunya. Una análisi des de l'Enfocament Segmentacionista de Cambridge per al cas de la comarca d' Osona. Universidad Autónoma de Barcelona.

Goerne, A. (2010). Diferencias y similitudes de la pobreza en el trabajo en Europa. Panorama Social, 12, 107-120.

Halleröd, B., Ekbrand, H., \& Bengtsson, M. (2015). In-work poverty and labour market trajectories: Poverty risks among the working population in 22 European countries. Journal of European Social Policy, 25(5). https://doi.org/10.1177/095 8928715608794

Heinz, J., \& Lund, F. (2012). Regimenes del bienestar y politica social: revisando el papel del trabajo y el empleo (Avances de Investigación No. 81).

Julián, D., y Hernández Aracena, J. (2017). Heterogeneidad estructural y precariedad laboral: Tomando en cuenta a los trabajadores pobres. Trabajo y Sociedad, 28 , 265-286.

Lohmann, H. (2009). Welfare States, Labour Market Institutions and the Working Poor: A Comparative Analysis of 20 European Countries. European Sociological Review, 25(4), 489-504. https://doi.org/10.1093/esr/jcn064

Lohmann, H., \& Marx, I. (2018). Handbook on In-Work Poverty. Edward Elgar Publishing. https://doi.org/10.4337/9781784715632

Lope, A. (2015). El trabajo y la inclusión social: una relación en peligro. Lavboratorio, 26, 107-129.

López-Roldán, P., \& Fachelli, S. (2017). Desigualdad y segmentación en los mercados del trabajo en España y Argentina. Anuario IET de Trabajo y Relaciones Laborales, 4, 15-33. https://doi.org/10.5565/rev/aiet.51

López-Roldán, P., Miguélez, F., Lope, A., y Coller, X. (1998). La segmentación laboral: hacia una tipología del ámbito productivo. Papers: Revista de Sociología, 55(4577).

López-Roldán, P., Semenza, R., \& Salvia, A. (2020). Comparing inequalities in the labour market from a segmentation perspective. In P. Lopez-Roldan \& S. Fachelli (Eds.), Towards a Comparative Analysis of Social Inequalities between Europe and Latin America. Springer.

Márquez, F. (2001). Trayectoria de Vida y Trabajo en Sujetos Pobres. Proposiciones, 32, 210-224.

Martínez, J. (2007). Regimenes del bienestar en América Latina (No. 11). Madrid: Fundación Carolina.

Marx, I., y Nolan, B. (2013). Trabajadores pobres. En O. Cantó (Ed.), Crisis, Desigualdad Económica y Mercado de Trabajo En España (pp. 99-118). 
MIDEPLAN. (1991). Evolucion de las Politicas Sociales en Chile 1920-1991.

Ministerio de Derechos Sociales y Agenda 2030. (2020). Ingreso mínimo vital. Retrieved October 19, 2020, from https://www.mscbs.gob.es/ssi/covid19/ingre soMinVital/home.htm

Ministerio de Sanidad, C. y B. S. (2020). Rentas mínimas de inserción. Retrieved October 19, 2020, from https://www.mscbs.gob.es/ssi/familiasInfancia/Servicio sSociales/RentasMinimas.htm

Montoya, A. (2014). Crisis económica y reforma laboral en España. In F. Cavas y J. Luján (Eds.), Crisis económica, reformas laborales y protección social: homenaje al profesor Jesús Maria Galiana Moreno (pp. 681-711). Murcia: Editum.

Narbona, K., Páez, A., y Tonelli, P. (2011). Precariedad laboral y modelo productivo en Chile (No. 1). Ideas para el buen vivir (Vol. 1). Santiago, Chile.

OECD. (2018). OECD Employment Outlook 2018. https://doi.org/10.1787/empl_ou tlook-2018-en

OIT. (2019). World employment and social outlook: Trends 2019. Geneva.

Paugam, S. (2007). Las formas elementales de la pobreza. Madrid: Alianza Editorial.

Pérez Ahumada, P. (2010). Clases sociales, sectores económicos y cambios en la estructura social chilena entre 1992 y 2013. Revista de La CEPAL, 126, 171-192.

Pérez, J. P. (2014). Mercados y bárbaros. San José: FLACSO.

Piketty, T. (2014). El capital en el S.XXI. México DF: Fondo de Cultura Econo\#mica.

Piovani, J. I., y Krawczyk, N. (2017). Los Estudios Comparativos: algunas notas históricas, epistemológicas y metodológicas. Educação \& Realidade, 42(3), 821840. https://doi.org/10.1590/2175-623667609

Recio, A. (2010). Capitalismo Español: la inevitable crisis de un modelo insostenible. Revista de Economía Crítica, 9, 198-222. https://doi.org/10.5152/akd.2010.173.

Santamaría, E. (2009). Trayectorias laborales en los márgenes del empleo: experiencias de precariedad en los procesos de construcción identitaria. Universidad del País Vasco.

Saraví, G. (Ed.). (2006). De la pobreza a la excusión: continuidades y rupturas de la cuestión social en América Latina. Buenos Aires: Prometeo Libros.

Sartori, G. (1994). Comparación y método comparativo. In G. Sartori y L. Morlino (Eds.), La comparación en las ciencias sociales. Mexico DF: Alianza Editorial.

Sen, A. (2000). Desarrollo y libertad. Buenos Aires: Planeta.

Sennett, R. (2007). La cultura del nuevo capitalismo. Barcelona: Anagrama.

Stiglitz, J. E. (2019). People, power and profits. Progressive capitalism for an age of discontent. London: Norton \& Company.

Tejero Pérez, A. (2018). Pobreza laboral en España. Un análisis dinámico. Revista Internacional de Sociologia, 76(2). https://doi.org/10.3989/ris.2018.76.2.16.54

Tonon, G. (2011). La utilización del método comparativo en estudios cualitativos en ciencia política y ciencias sociales: diseño y desarrollo de una tesis doctoral. Kairos. Revista de Temas Sociales, 27, 1-12.

\section{Notas}

1 Este artículo se ha elaborado en el contexto del proyecto DINAMOS1 (Referencia PID2019-106548GB-C21): Movilidad social intrageneracional y trayectorias ocupacionales segmentadas, financiado por el Ministerio de Ciencia e Innovación de España y coordinado por el Centro de Estudios Sociológicos sobre la Vida Cotidiana y el Trabajo de la Universitat Autònoma de Barcelona, siendo el investigador 
principal el Dr. Pedro-López-Roldán (https://pagines.uab.cat/dinamos/). El Autor, José Zawadsky, es doctorando en Sociología, Universitat Autònoma de Barcelona, Becario Becas Chile.

2 Paugam llama descalificación social al "proceso de expulsión del mercado de trabajo de franjas cada vez más numerosas de la población y las experiencias vividas de la relación de asistencia que acompaña las diferentes fases" (Paugam, 2007, p. 66). 